\title{
OBSERVATION OF THE SOLAR SOFT X-RAY COMPONENT; \\ STUDY OF ITS RELATION TO TRANSIENT AND SLOWLY- \\ VARYING PHENOMENA OBSERVED AT OTHER
}

WAVEI.ENGTHS

\author{
RICHARD G. TESKE \\ Dept. of Astronomy and the McMath-Hulbert Observatory, \\ The University of Michigan, Ann Arbor, Mich., U.S.A.
}

(Received 8 July, 1968)

\begin{abstract}
Alstract. Solar X-rays from $8.12 \AA$ have been observed wilh an ion chamber photometer and fluxes derived from the observations after an assumption concerning the spectral distribution. The time variation of the X-ray flux correlates well with the radio flux, plage index, and sunspot number. Comparisons of $\mathrm{X}$-ray and optical events are given; flares seem to produce soft $\mathrm{X}$-rays, but some soft X-ray bursts are apparently not associated with flares. The total encrgy involved in the soft $X$-ray bursts may be a significant amount of the tolal flare radiation.
\end{abstract}

\section{Instrumentation}

The Michigan soft X-ray ion chamber photometer is located in the wheel of OSO-III. The ion chamber is filled with about one atmosphere of dry nitrogen gas and has a 5-mil thickness aluminum foil window; the efficiency of response as a function of wavelength for such an ion chamber has been published (ACTON et al., 1963). The detector responds principally to energy in the wavelength range $8-12 \AA$, though there is also a low elficiency of response between 2-5 $\AA$. In OSO-IIJ, the cadence of mainframe telemetry rate and wheel rotation rate results in roughly one word of solar soft $X$-ray data and two words of particle background data for each period of wheel rotation.

Data generated by the instrument are converted to energy fluxes $E(8,12)$ ergs $\mathrm{cm}^{-2} \mathrm{sec}^{-1}$ for the wavelength band $8-12 \AA$ under the assumption that the radiation is distributed as in a black-body curve for $2 \times 10^{6} \mathrm{~K}$ (KRI:PLIN, 1961). If the slope of the actual flux-distribution curve departs from the assumed one (e.g., NI:UPERT et al., 1967; Fritz et al., 1967; RUGge and W ALKER, 1967), the true encrgy flux may be approximately recovered by applying a correction factor (Figure 1). Attempts are being made to utilize observations obtained during occultations of the sun behind earth's atmosphere near satellite dawn and twilight to determine spectral slopes for the flux distribution which may be appropriately applicd to this experiment.

Two automatically-selected ranges of operation are incorporated into the instrument, one with a high dynamic sensilivity $(3400 / 1)$ and one with a lower dynamic sensitivity $(125 / 1)$ covering a total flux range $0<E(8,12)<0.12$. The instrument has been saturated by the peak emission from great flares. An elcctrical calibration occurs about every $6 \mathrm{~min}$, and has shown that the instrumental cnergy scale has been 
stable to about $1 \%$ since launch. The absolute calibration is probably within $6 \%$ of correct.

Reliability of operation may be verified by using the sun itself as a standard source. There exists a good statistical - and physical - relationship between the $2800 \mathrm{MHz}$ solar flux and the soft X-ray flux (e.g., Figures 2 and 3). Comparison of these two fluxes for $\Lambda$ pril 1967 and for August 1967 separately gave very closely the same relationship. Over that 5-month interval, at least, no changes in ion-chamber response occurred.

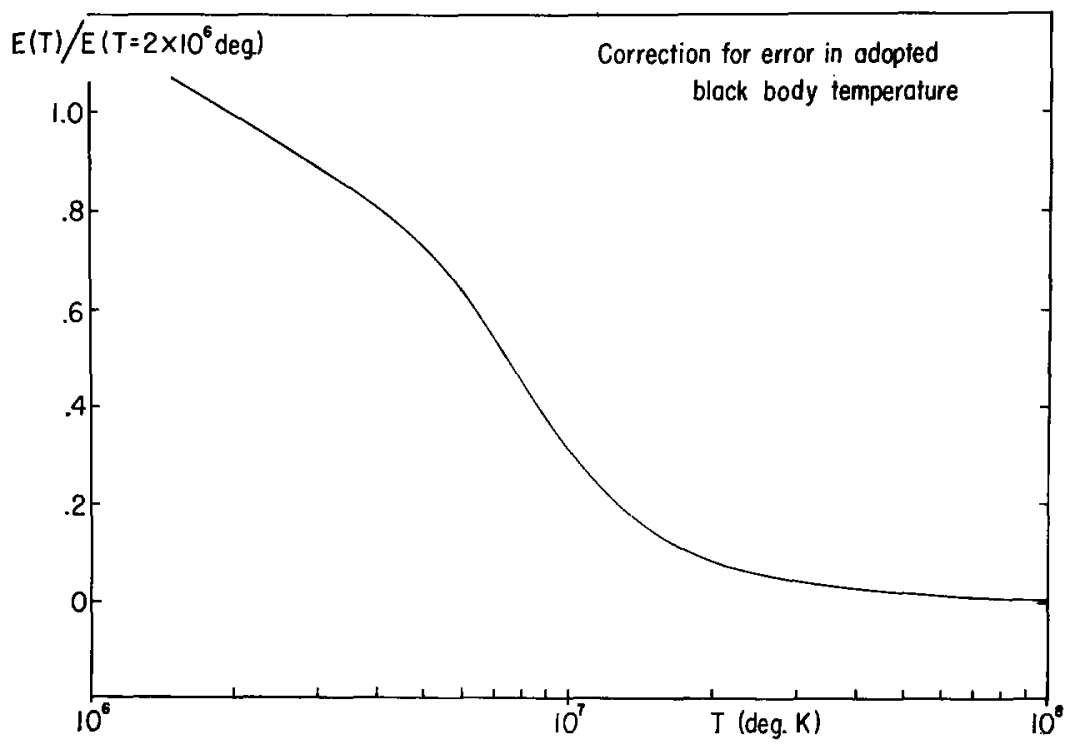

Fig. 1. If the truc solar flux distribution with wavclength approximatcs a black-body curve for temperature $T$ (abscissa), energy fluxes quoted in this paper must be multiplied by the value of the ordinate.

\section{Observations of the Non-Flaring Sun}

The slow variation of soft X-ray flux with solar activity is shown in Figure 2 for the period March through August 1967. The index which is here used to characterize the quict X-ray sun is the daily basc-level, which is the lowest known flux reached during the day and is thus an index of the non-flaring sun. The X-ray base-levels are still preliminary, since complete 24-hour data have not yet been analyzed for the full six months.

As may be judged from Figure 2, the soft X-ray daily base-level correlates well with other indices of the general level of solar activity: the $2800 \mathrm{MHz}$ daily solar flux $(\rho=0.82)$, a McMath-Hulbert Observatory plage index $(\rho=0.72)$ and the Zürich sunspot number $(\rho=0.78)$. Hence, the major component of the soft X-radiation bcing obscrved ariscs in the active centers (cf. UNDER wOOD and MUNEY, 1967). The 
base-level enhancements of August 18/19 and of August 30/31, apparently uncorrelated with the other solar indices, coincide with East and West limb passages of a major active center on the sun (McMath plage region 8942) and apparently show it to have been strongly limb-brightened in X-radiation.

In addition to its correlation with the $2800 \mathrm{MHz}$. flux density, the soft X-ray background changes in a predictable way with radio flux density at other frequencies

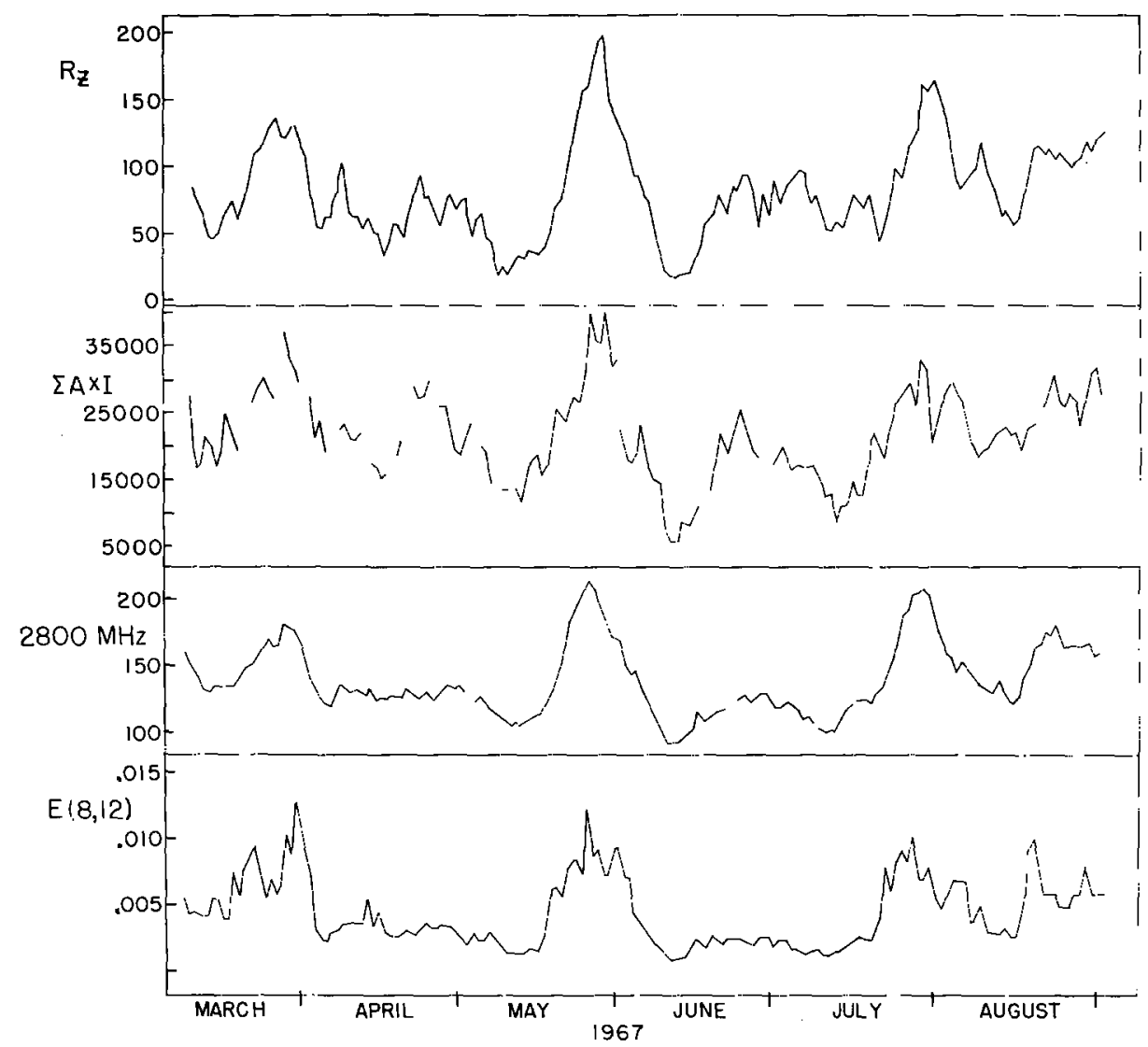

Fig. 2. Soft X-ray base level fluxes (bottom) are compared with the $2800 \mathrm{MHz}$ daily flux density, with a plage index which is the sum of products of plage areas by excess intensity in the CaII $\mathrm{K}$ line core, and with the Zürich daily sunspot number (top).

(Figure 3). For a given variation $\Delta E(8,12)$ of soft $\mathrm{X}$-ray flux, the variation of radio flux density is greater at $2800 \mathrm{MHz}$, and is less at higher and lower rrequencies. A linear fit scems adequate for the data so far cxamined. When the data for Figure 3 were assembled, no account was taken of possible center-limb effects.

Figure 4 contains data for four days which are representative of a range of solar activity conditions. In the figure, each point represents a 67 -second time-average of 
$\mathrm{X}$-ray flux. Vertical bars represent satellite night, which is about $35 \mathrm{~min}$ long. Satellite day is about an hour long. Times which are given, so as to provide a time-scale, refer to the time of a satellite dawn. For May 11, May 5, and June 1, times are discontinuous across satellite night, while for April 15 the time is continuous.

On May 11 quiet solar conditions prevailed: the daily $2800 \mathrm{MHz}$ flux density of 104.0 was lowest since late December 1966 and no regions of significant activity were present on the visible solar hemisphere. These quiet conditions are reflected in a nearly flat, uniform X-ray flux-time curve. Arrows indicate the reported starting times for two subflares. We have associated the nearby X-ray enhancements with those flares.

In contrast, on May 5 the X-ray flux-time curve was constantly disturbed, showing significant variations throughout the period of time depicted. On this date a flare-rich plage region (No. 8791) was located in the Southwestern quadrant of the disk, and

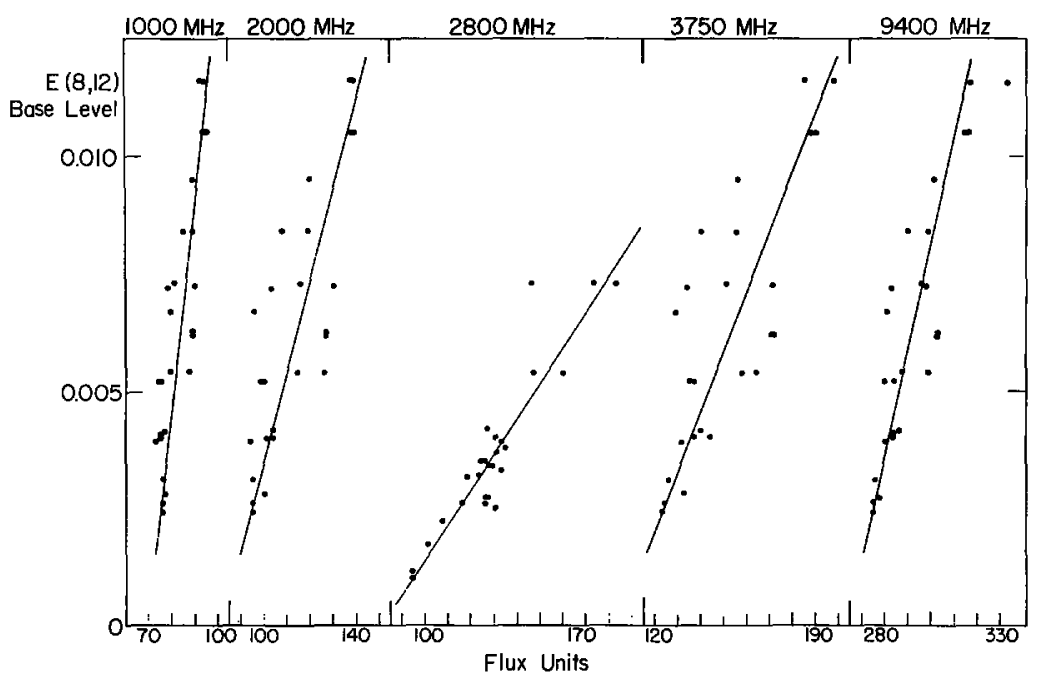

Fig. 3. Radio flux densities at $2800 \mathrm{MHz}$ are those reported by Algonquin Radio Observatory (Ottawa) and at other frequencies are those reported by the Rcsearch Institute of Atmospherics at Nagoya University (Toyokawa). Radio and X-ray fluxes wcre observed simultaneously when no flares were occurring. The Toyokawa and Ottawa data represent samples during different months. One flux unit equals $10{ }^{23} \mathrm{~W} \mathrm{~m}^{-2} \mathrm{~Hz}^{-1}$.

the $2800 \mathrm{MHz}$ daily flux density was 125.8 . Arrows point to enhancements which we have associated with subflares reported during our X-ray observing hours on May 5.

The flux-time curve for A pril 15 again refers to moderately active solar conditions, when the $2800 \mathrm{MHz}$ flux stood at 123.2. It is inserted in Figure 4 to demonstrate operation of the instrument as it switches from its range of higher dynamic sensitivity to its range of lower sensitivity. The major enhancement in the diagram accompanied a reported subflare. The second enhancement was not accompanied by a reported 
optical event. We have tentatively associated it with a small cvent in plage No. 8760 on the Northwest limb, not seen very clearly on our flare films. There occurred a weak brightening of the plage, and a surge-like, faint feature was seen projecting above the limb. Times of beginning, maximum and end of the event coincide in time with the $\mathrm{X}$-ray increase.

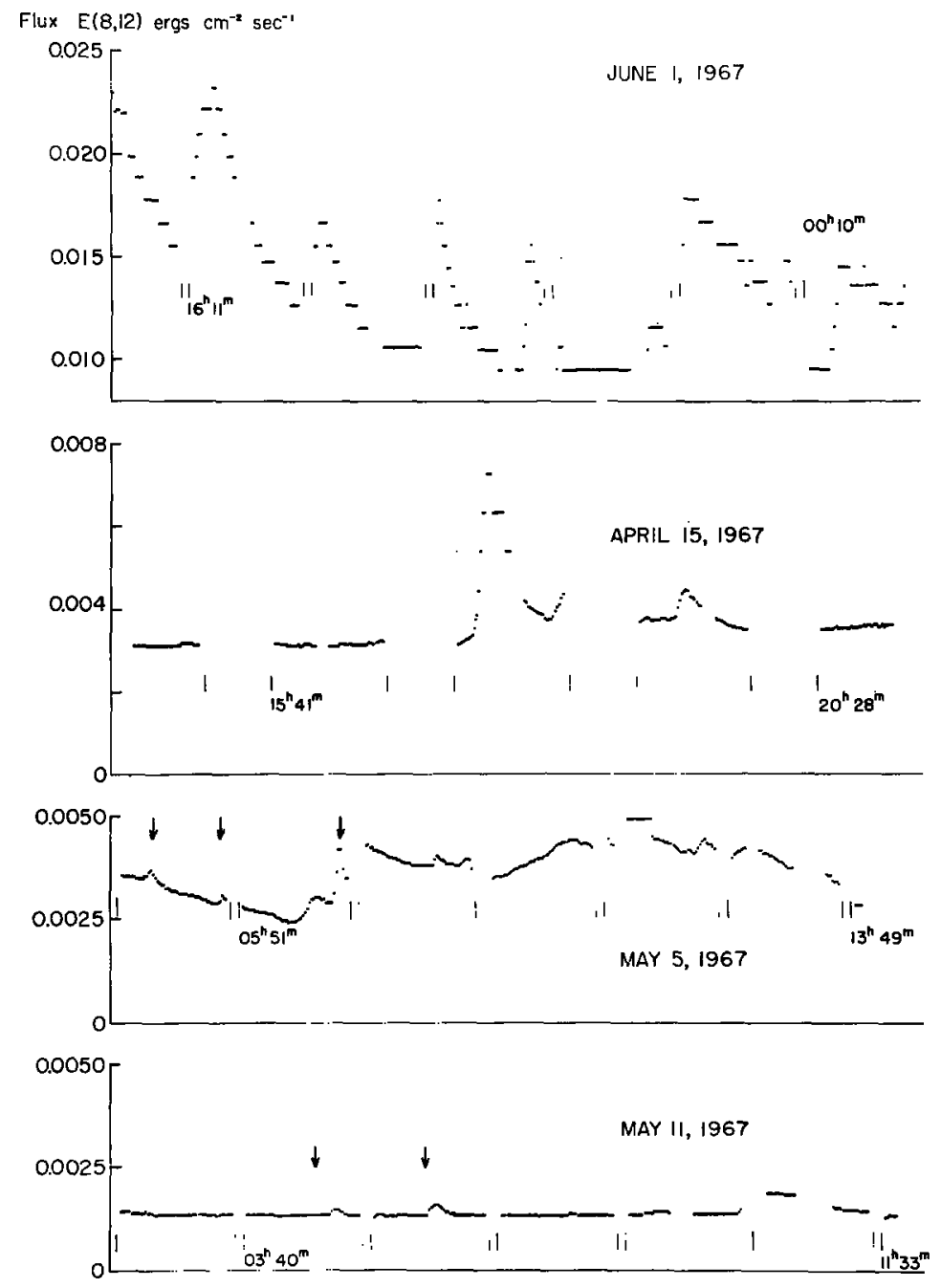

Fig. 4. Representative data for four days in 1967.

At times of great solar activity, such as on June 1, the solar soft X-ray flux varies almost constantly through a great range of flux values. On that date the $2800 \mathrm{MHz}$ flux was 169.7. McMath plage region No. 8831, then in the Northeast quadrant of the disk, was at its peak of flare activity on June 1 and June 2. Enhancements of 
$X$-ray flux seen in our record for June 1 accompanied subflares and flares of importance 1. X-ray flux increases which accompanied subflares on June 1 were generally greater than those accompanying subllares on the olher dates in Figure 4.

Flare associated X-ray enhancements will be discussed in the next section.

Our data show that during times when no reportable optical events occur on the sun, the solar soft $X$-ray flux may still undergo some variations. Somelimes these non-flare X-ray fluctuations can be identified with fluctuations in $\mathrm{H} \alpha$ plage intensity and with events at the limb, but often there is no clearly-associated optical $\mathrm{H} x$ countcrpart. At times of relatively high solar activity, as evidenced by a relatively high X-ray base-level or by a relatively high $2800 \mathrm{MHz}$ flux, these non-flare X-ray fluctuations occur frequently, with an amplitude of $\approx 10 \%$ or more of the average flux level, and on a time scale of tens of minutes. As solar activity moderates, the $X$-ray fluctuations also moderate as to their amplitude and frequency of occurrence. At the quietest times seen so far, the X-ray flux-time curve becomes smooth, showing only occasional fuctuations of very small amplitude.

\section{Observations of Active Events}

The relationship of soft X-ray fux enhancements to transient solar events observed optically and at radio wavelengths is being more clearly outlined by the OSO-III data. As regards flares, the times of begioning, maximum and ending of the X-ray events, as well as the total flux cnhancement, appear to be statistically related to the timing of the optical event and to its importance classification. (See, however, the discussion by UNDERWOOD (1968).)

We have attempted to determine the frequency with which $\mathrm{X}$-radiation accompanies flares. The study has been limited to those hours during each day for which we have already reduced data.

In a sample of 57 subflares reported on a world-wide basis, 51 were definitely accompanied by X-rays. Of 47 subflares which were observed cinematographically at the McMath-Hulbert Observatory during March, April and May of 1967, 44 were definitely accompanied by soft X-rays. The three 'failures' occurred when our X-ray detector was operating in its range of lower sensitivity. If the subllare sample is confined to those subflares observed cinematographically at McMath during hours when the $X$-ray detector was operating in its higher sensitivity range, then it appcars that all the subflares werc associated with X-ray emission, although at times it was very weak emission.

Only six flares of importance 1 were observed cinematographically at the McMath Hulbert Observatory between March 10 and June 30 and during hours for which we already have reduced data. Each was accompanied by a significant $X$-ray increase. Only 23 out of 26 importance-1 flares reported on a world-wide basis during hours for which we have reduced data from March 10 through May 31, had X-rays with them. None of the three 'failures' was reported by more than one observing station, however, and they may be accidental reports. 
Hence, we are of the opinion that probably all flares are the site of emission of soft X-rays, although at times the emission is indeed minor (Figure 4). In order for a flare-associated enhancement to be recognizable in our data as reduced so far, its duration and amplitude must be such as to yicld a time-integrated flux between 8 and $12 \AA$ of a lew times $10^{25}$ ergs at the sun, assuming emission into $4 \pi$ steradians and no photon scattering.

On the other hand, we have asked with what frequency visible flares or other active events accompany X-ray bursts. In a sample of 79 flux enhancements of all sizes recorded during March, April and May of 1967, only 57 bursts could be identified with flares reported on a world-wide basis and four with limb events such as surges leaving 18 of $\mathrm{thcm}$, or $23 \%$ which could not be identified with reported optical phenomena. More than half of these remaining bursts occurred during European observing hours, where subflares are usually not reported as a matter of course. The average flux enhancement for the unidentified bursts was smaller than the average flux enhancement accompanying reported subflares in the same sample.

Time-lapse filtroheliographic flare patrol films made at the McMath-Hulbert Observatory have been examined in connection with some of the preliminary X-ray data. The total film examined is about equivalent to 35 days of full-time day-light flare patrol. Two relatively large X-ray bursts have been. found which are not clearly associated with visible flare activity; both are somewhat smaller than the enhancement usually associated with flares of importance 1. One of these may have possibly been associated with activity behind the Southwest limb (July 11). The second took place near the time of a subflare on the disk, but is in very poor time-association with the H $x$ event (July 29).

Studies of individual flare events in connection with the X-ray data have permitted us to draw some tentative generalizations which will be investigated further as more data are reduced. Sources for the studies include the McMath-Hulbert flare patrol films, the ESS $\Lambda$ Solar-Geophysical Data Bulletin, HAO Preliminary Reports, flare and SID lists from McMath and Manila and radio data from Toyokawa, Hiraiso, Sagamore Hill and Ottawa.

X-ray enhancements associated with flares appear to be fair guides to flare importance in the sense that subflares tend to be associated with smaller enhancements than flares of importance 1 , and so on, yet flares within a given importance class may give rise to a greal range of $\mathrm{X}$-ray flux.

Generally the $F \propto$ flare and the X-ray event begin at about the same time. The $\mathrm{H} \varkappa$ intensity peak will most frequently precede or coincide with the X-ray maximum. In about $20 \%$ of flares the $\mathrm{H} \alpha$ maximum intensity follows the X-ray peak. In flares of importance $1^{-}$and 1 the $\mathrm{H} \alpha$ and X-ray events tend to end together, while in major flares the X-rays tend to outlast the visible event for a length of time which depends upon flare importance. Following the great flares of March 22 and May 23, 1967, the soft X-ray flux remained above pre-flare levels for at least five hours. Many exceptions to these generalizations have been observed. For example, somctimes the visible flare will begin two or three min before the X-ray flux begins to increase (Figure 5). Again, 
following the importance-2 flare of May 6, 1967, the soft X-ray flux returned to its pre-flare level quite precisely and almost at once after the flare was reported to have ended. Flare events occurring close to the sun's limb sometimes demonstrate the greatest departures from these generalizations, an effect which is probably connected with the well-known decreased visibility and shorter duration of flares near the limb.

Examples of X-ray bursts accompanying flares of importance $\mathrm{I}$ and 2 are shown in Figures 6 and 7, together with photometric $\mathrm{H} x$ intensity curves (intensity expressed in arbitrary units) and the accompanying $\mathrm{cm}-2$. bursts. In both cvents a slow brightening in both $\mathrm{H} x$ and $\mathrm{X}$-rays preceded the $\mathrm{cm}-\lambda$. burst. At that time the flares abruptly brightened in $\mathrm{H} \alpha$ and $\mathrm{X}$-rays.

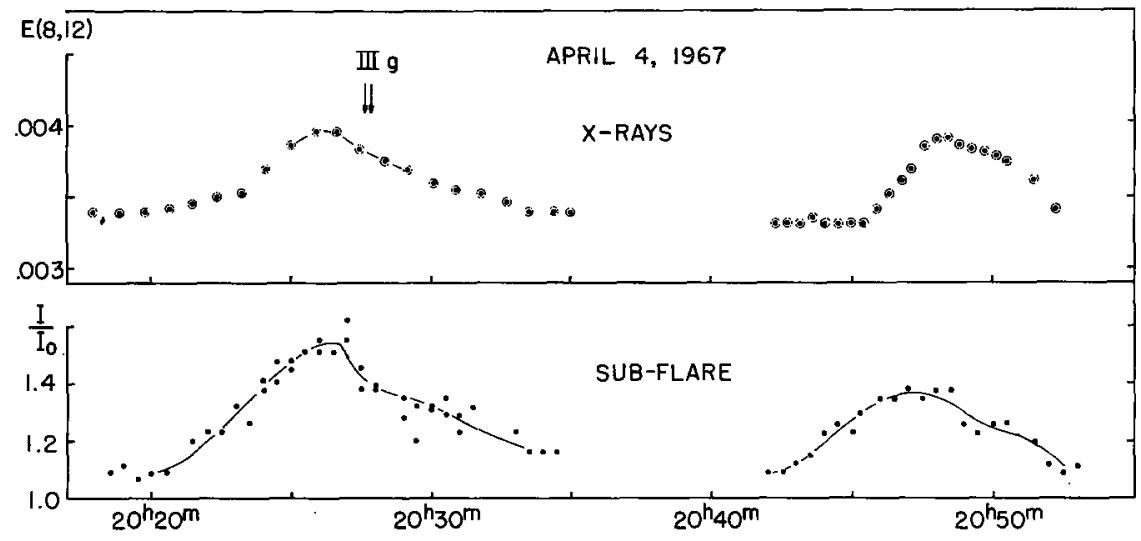

Fig. 5. Photometric $\mathrm{Ha}$ intensity curves for two subflares (lower) and the associated X-ray flux enhancements. The sun was occulted behind earth's atmosphere beginning at about $20^{\mathrm{h}} 51^{\mathrm{m}}$. The first subflare (S20E22) was associated with a great ejection of dark material, the second (S25E50) was not.

In the July 25, 1967 cvent (Figure 6), the importance-1 flare lasted until after $15^{\mathrm{h}} 00^{\mathrm{m}}$, although the $\mathrm{H} x$ light curve has not been carried along that far. The subflare which began at $14^{\mathrm{h}} 50^{\mathrm{m}}$ on that date did not affect the X-ray record probably because our instrument was then operating in its 'flare mode' of low dynamic sensitivity, in which the weak emission accompanying subflares is often not detected (cf. Figure 5 , where operation was in the higher sensitivity mode).

While the $\mathrm{H} \alpha$ and $\mathrm{X}$-ray curves often appear qualitatively similar, as in Figures 6 and 7 , the structure of the soft X-ray burst is usually very different from the structure of the accompanying $\mathrm{cm}-\lambda$ burst. Typically, the maximum of the $\mathrm{cm}-\lambda$. burst occurs some minutes prior to the $\mathrm{X}$-ray maximum $(\approx 80 \%)$, more rarely the 1 wo maxima coincide within a minute $(\approx 15 \%)$ and in still rarcr cases a weak $\mathrm{cm}-\lambda$ maximum occurs after the soft $X$-ray peak. Thus, the $X$-ray maximum tends to associate itself most clearly with the post-burst phase of the radio cmission at centimeter wavclengths. On the other hand, there is a strong tendency for the soft $X$-rays to begin their increase earlicr than the reported beginning of the $\mathrm{cm}-\hat{\lambda}$ burst. 
This latter effect is suggestive of physical conditions in the flare volume, to which the mechanism that gives rise to the soft X-radiation is more sensitive than is the mechanism that generates $\mathrm{cm}-\lambda$ radiation. In flares, an increasc of $2800 \mathrm{MHz}$ flux density by 5 flux units $\left(5 \times 10^{-22} \mathrm{~W} \mathrm{~m}^{-2} \mathrm{~Hz}^{-1}\right)$ is accompanied by an X-ray increment $\Delta E(8,12)$ of $0.005 \mathrm{ergs} \mathrm{cm}^{-2} \mathrm{sec}^{-1}$ (Figure 8). The relationship in the slowly-varying component (Figure 3) is such that an increase of 70 flux units at $2800 \mathrm{MH}$. accompanies an increment in X-ray flux of $\Delta E(8,12)$ of $0.005 \mathrm{ergs} \mathrm{cm}^{-2} \mathrm{sec}^{-1}$. Whrre (1964) noted a similar sensitivity in the $2-8 \AA$ flux.

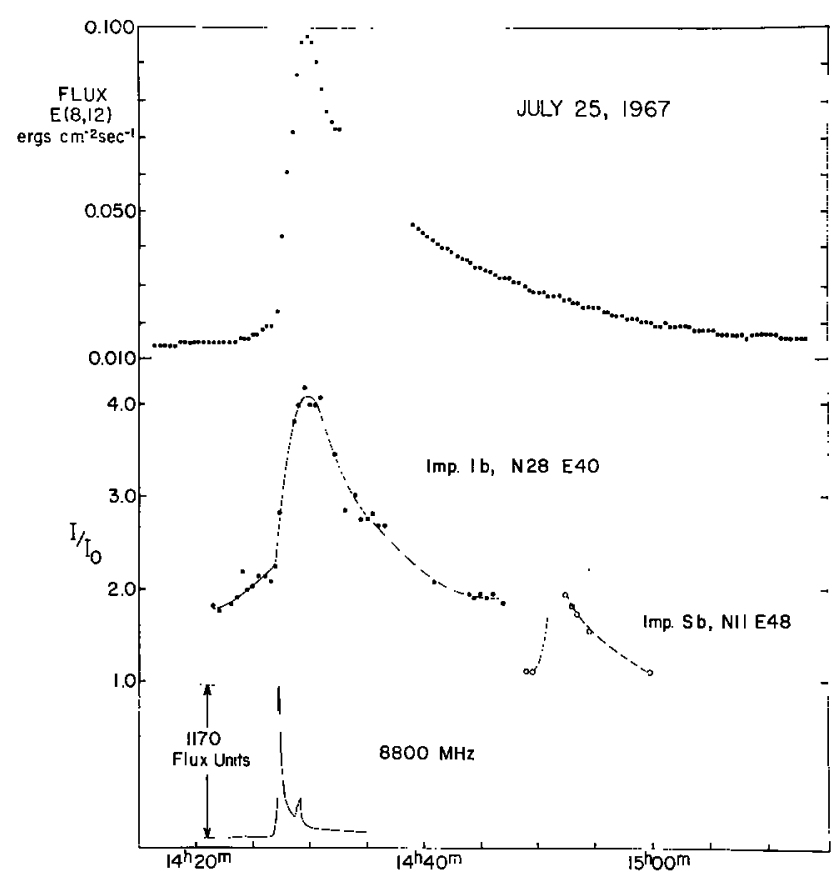

Fig. 6. The gap in the X-ray record (top) occurred during tape-recorder playback. Clouds interfered with the $\mathrm{H} x$ flare observations (center). The $8800 \mathrm{MH}$. record was reported by Sagamore Hill (lower).

\section{X-Ray Energy Emitted by Flares}

Information concerning physical conditions in flares may be obtained through knowledge of the energy invested by flares in various parts of the electromagnetic spectrum and in particle and plasma emissions. Further, the total energy emitted by flares and the time scale for its release are consiraints upon possible flare mechanisms. Thus, it is of interest to obtain from our data some indication of the total energy emitted as soft X-rays.

Because the spectral distribution of $\mathrm{X}$-radiation is a function of time during a flare event (RUGGe and WALKI:R, 1967; CULHANE et al., 1963), time-integrals of flare 
encrgy between 8 and $12 \AA$ are uncertain, and it is difficult to estimate at this time what the uncertainty is. It may not be overly pessimistic to estimate crrors by up to a factor of 3 for ordinary flares, and by up to a factor of 5 for flares of importance 2 and 3 , in the sense that the measured total energies may be too high.

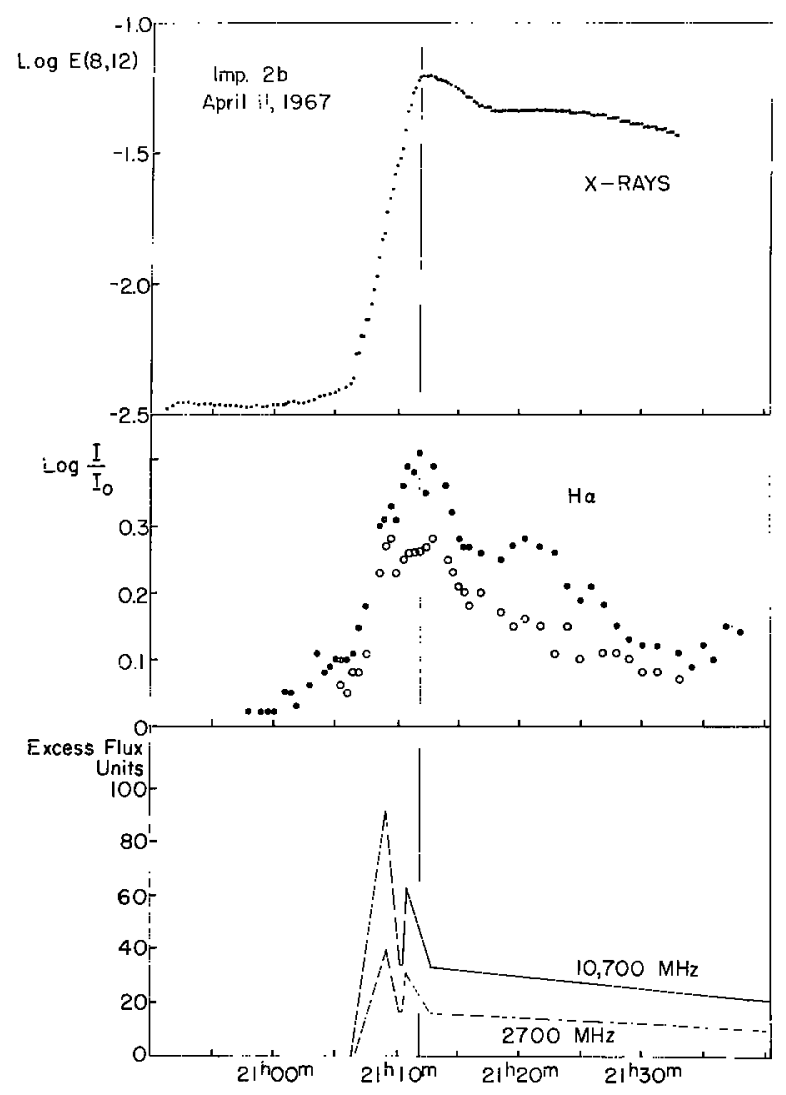

Fig. 7. The importance-2b flare took place at $\mathbf{2} 23 \mathrm{~W} 72$. $1 \mathrm{H}$ intensities measured in two parts of the flare are shown on a logarithmic scale, as are the $X$-ray fluxes. The $\mathrm{cm}-\hat{\lambda}$ event is drawn schematically for two frequencics according to author's interpretation of data in ESSA Solar-Gcophysical Data Bulletin.

TABLE: I

Total Flare Energies $\int E(8,12) \mathrm{d} t$

\begin{tabular}{|c|c|c|c|c|c|}
\hline $\begin{array}{l}\text { Date } \\
\text { (1967) }\end{array}$ & Importance & $\begin{array}{l}\text { No. of } \\
\text { Flares }\end{array}$ & $\begin{array}{c}\text { Mean Energy } \\
\text { (ergs) }\end{array}$ & & $\begin{array}{l}\text { Range } \\
\text { (crgs) }\end{array}$ \\
\hline - & $1-$ & 8 & $3 \times 10^{2 \overline{7}}$ & $7 \times 10^{25}$ & $-8 \times 10^{27}$ \\
\hline$\ldots$ & 1 & 8 & $6 \times 10^{28}$ & $1 \times 10^{28}$ & $-2 \times 10^{29}$ \\
\hline March 22 & $3^{-}$ & 1 & $\approx 10^{30}$ (Total) & & \\
\hline May 23 & $2,2,2,2$ & 4 & $>5 \times 10^{30}($ Total $)$ & & - \\
\hline
\end{tabular}




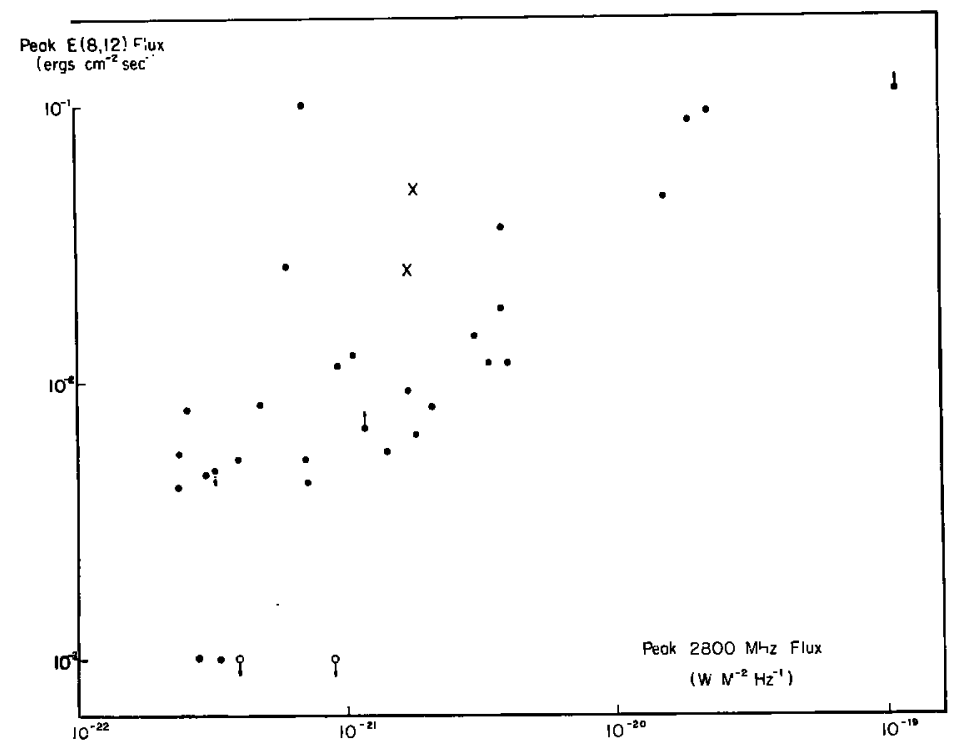

Fig. 8. Relation of peak fluxes for associated X-ray and $\mathrm{cm}-i$ events. Filled circles: simple bursts with flares; crosses: complex bursts with flares; open circles: simple bursts without reported flares. All values are as measured at the earlh, uncorrected for earth's orbital eccentricity.

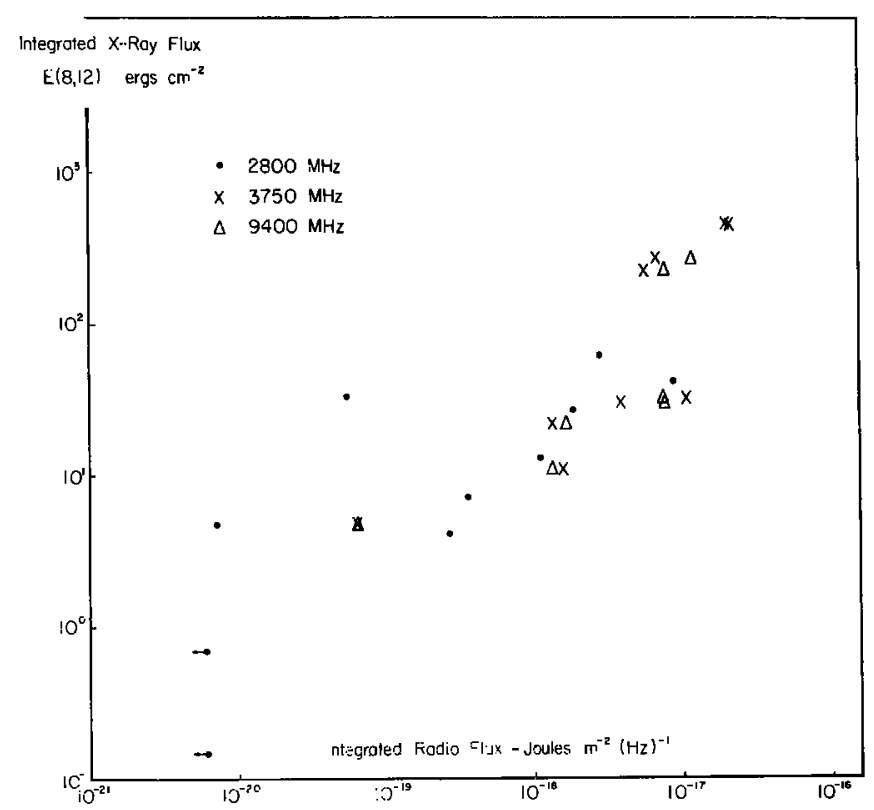

Fig. 9. Relation of time-integrals of $\mathrm{X}$-ray and cm- $\lambda$ events. The $2800 \mathrm{MHz}$ point at $5.5 \times 10^{-20}$ Joules $\mathrm{m}^{-2} \mathrm{~Hz}^{-1}$ represents an event on July 11 which was not clearly flare-associaled. The two $2800 \mathrm{MHz}$ points towards lower left represent estimated upper limits based upon no report during observing hours. All values as measured at earth, uncorrected for earth's orbital cccentricity. 
We have assumed that the flare radiation is relcased into $4 \pi$ steradians and that none of the inwardly-directed photons are scattered. Time-integrals of cnergy measured by us in a number of flares, and computed for the wavelength band 8-12 are summarized in Table I. The estimates in the table should be viewed as upper limits.

The energy radiated by flares at $\mathrm{cm}$-wavelengths is related to the soft $X$-ray energy in the sense that large X-ray events tend to accompany large $\mathrm{cm}-\lambda$ events and vice versa. Peak X-ray flux and peak cm- flux density in associated events are plotted in Figure 8. We note that the $X$-ray peak is still high enough at a radio flux density peak of 1 or 2 flux units to be clearly discernable in our data. It appears likely that even smaller X-ray bursts than those investigated may be accompanied by unreportably small radio bursts.

When time-integrals of energy at $\mathrm{cm}$ - and X-ray wavelengths are examined, we find again that there is a strong tendency for the greater $X$-ray events to accompany the greater radio events (Figure 9). Time-integrals of radio flux density in Figure 9 were obtained by multiplying tabulated mean fluxes by tabulated durations. ARNOLDY et al. (1967) have shown that a definite relation like that suggested by Figure 9 exists for the 10-50 kV X-rays. Further study of our data will be necessary before quantitative relations can be confidently established with them.

\section{References}

Acton, L. W., Chubb, T. A., Kreplin, R. W., and Mfackins, J. F.: 1963, J. Geophys. Res. 68, 3335. ArNoldy, R. L., KAnf, S. R., and Winckler, J. R.: 1967, Solar Phys. 2, 171.

Culmane, J. A., Will.more, A. P., Pounds, K. A., and Sanrord, P. W.: 1963, Space Res. IV, 741. Frit7, G., Kreplin, R. W., Meekins, J. F., Unzicker, A. E., and Frifdman, H.: 1967, Astrophys. J. 148, L133.

KrePLiN, R. W.: 1961, Ann. Geophys. 17, 151.

Neuperi', W. M., GAtes, W. J., Swartz, M., and Young, R.: 1967, Astrophys. J. 149, L79.

RugGie, H. R. and Walkfr, A. B. C., Jr.: 1967, Preprint (Submitted to Space Res. VIII).

UNDERWOOD, J. H.: 1968, Science 159, 383.

UNderwood, J. H. and MUNty, W. S.: 1967, Solar Phys. 1, 129.

Whirl:, W. A.: 1964, in AAS-NASA Symposium on the Physics of Solar Flares (ed. by W. Hess), (NASA SP-50), p. 131. 